\title{
Lung Ultrasound for Detection of Pneumothorax in Neonates
}

\author{
Anup Thakur $^{1} \cdot$ Neelam Kler ${ }^{1} \cdot$ Pankaj Garg ${ }^{1}$
}

Received: 1 April 2019 / Accepted: 8 July 2019 / Published online: 19 July 2019

(C) Dr. K C Chaudhuri Foundation 2019

To the Editor: Lung ultrasound (USG) has been well studied for diagnosis of pneumothorax in adult emergency care; however limited data exists on its application in critically ill neonates $[1,2]$. We report two cases of pneumothorax detected by lung USG in suddenly decompensating neonates.

Our first case was a term infant with birth weight $2590 \mathrm{~g}$, diagnosed to have esophageal atresia with tracheo-esophageal fistula. He underwent a right-posterolateral thoracotomy with primary repair and ligation of fistula on day 1 of life. Postoperatively, he was mechanically ventilated but on day 2 , he had sudden deterioration with increase in oxygen requirement from 0.25 to 0.70 . Transillumination was positive on the right side and corresponding lung USG done (using Sonosite M Turbo machine with 13-6 MHz linear probe) at three standardized BLUE points [3] showed classical signs of pneumothorax in the form of multiple A lines, absent B lines, absent lung sliding, Stratosphere sign in M mode and lung point [3]. Chest radiograph confirmed the presence of pneumothorax.

Our second case was an infant, born at $36 \mathrm{wk}$ with a birth weight $2500 \mathrm{~g}$, who developed respiratory distress soon after birth requiring continuous positive airway pressure. At $5 \mathrm{~h}$ of age, he deteriorated in the form of worsening respiratory distress and signs of poor perfusion, requiring mechanical ventilation and inotropes. Transillumination was negative. Chest radiograph showed a small left sided pneumothorax which was missed by the resident on call. Corresponding lung USG showed classical signs of pneumothorax as described above. Chest X-ray cross table lateral view later confirmed presence of small pneumothorax. Thoracocentesis was performed following which he improved and repeat chest radiograph showed resolution of pneumothorax.

Anup Thakur

dr.thakuranup@gmail.com

1 Department of Neonatology, Institute of Child Health, Sir Ganga Ram Hospital, Rajinder Nagar, New Delhi 110060, India
Lichtenstein et al. had described the classical findings of pneumothorax. In normal lung, lung sliding sign due to sliding of the visceral pleura against the parietal pleura is seen in B mode as sparkling below the pleural line and in the M mode, as a sea shore sign. In suspected pneumothorax, one should first detect abolished lung sliding and that translates into the standardized Stratosphere sign in the M mode. The next step then is to visualize $A$ line sign and a negative search for B lines in the field. This is called A ' profile and suggests pneumothorax. Presence of even a single B-line rules out pneumothorax. Only when this is established, the next sign sought for, which is pathognomonic for a pneumothorax is the lung point. This is the junction between pneumothorax and the lung, when sudden transition from abolished lung sliding to presence of lung sliding appears $[4,5]$. Both our cases had all classical signs described above, irrespective of the fact that in one of them the pneumothorax was not found on transillumination and nearly missed on chest radiograph.

\section{Compliance with Ethical Standards}

Conflict of Interest None

\section{References}

1. Raimondi F, Rodriguez Fanjul J, Aversa S, et al; Lung Ultrasound in the Crashing Infant (LUCI) Protocol Study Group. Lung ultrasound for diagnosing pneumothorax in the critically ill neonate. J Pediatr. 2016;175:74-8.e1.

2. Liu J. Lung ultrasonography for the diagnosis of neonatal lung disease. J Matern Fetal Neonatal Med. 2014;27:856-61.

3. Lichtenstein DA, Mezière GA. The BLUE-points: three standardized points used in the BLUE-protocol for ultrasound assessment of the lung in acute respiratory failure. Crit Ultrasound J. 2011;3:66.

4. Lichtenstein D. Lung ultrasound in the critically ill. Curr Opin Crit Care. 2014;20:315-22.

5. Lichtenstein DA, Mezière G, Lascols N, et al; Ultrasound diagnosis of occult pneumothorax. Crit Care Med. 2005;33:1231-8.

Publisher's Note Springer Nature remains neutral with regard to jurisdictional claims in published maps and institutional affiliations. 